\title{
DNA Binding Agent
}

National Cancer Institute

\section{Source}

National Cancer Institute. DNA Binding Agent. NCI Thesaurus. Code C2842.

DNA binding molecules regulate mechanisms central to cellular function, including DNA replication and gene expression. Many small molecules that mimic or block these processes offer potential therapeutic agents. Understanding how complexation affects both the structural and mechanical properties of DNA is an important step towards understanding the functional mechanisms of binding agents, and may also provide a key to more rational drug design. There are three major classes of binding modes: intercalation, groove binding and covalent crosslinking. 